\title{
Neuro-Fuzzy Supervised Training Algorithm for Varied Chicken Disease Recognition
}

\author{
${ }^{1}$ Imianvan A.A. and ${ }^{2}$ Obi J.C. \\ Department of Computer Science, University of Benin, Benin City. Nigeria \\ ${ }^{1}$ tonyvanni@yahoo.com; 2tripplejo2k2@yahoo.com
}

\begin{abstract}
Poultry farming is an integral part of human existence. It link or interlink most, if not all of human endeavors which provides food in terms of meat and other proteins ingredients for human consumption and existence. Diseases on the other hand, are usually a resultant of human interaction with the ecosystem which has affected poultry farming for centuries. Most of the approaches applied in chicken disease recognition is subjective (based on the experiences, skills, exposure and talents of a personnel) in nature at best. Fuzzy Supervised Neural Network Training Algorithm has been designed and implemented with Matrix Laboratory (MATLAB) and Hypertext Pre-processor as the simulation tools and language respectively. This paper demonstrates the practical application of algorithm techniques in medical the agricultural sector with the aim of distinctive recognition of four classes of chicken disease.
\end{abstract}

Keywords: chicken disease, Fuzzy set, Fuzzy Logic, Algorithm, Supervised-Neural-Network

\section{Introduction}

Poultry farming is the raising of domesticated birds such as chickens, turkeys, ducks, and geese, for the purpose of farming meat or eggs for food. Poultry are farmed in great numbers with chickens being the most numerous. More than 50 billion chickens are raised annually as a source of food, for both their meat and their eggs (Sherwin et al., 2010). Chickens raised for eggs are usually called layers while chickens raised for meat are often called broilers (Grandin and Johnson, 2005).

Commercial hens usually begin laying eggs at 16-20 weeks of age, although production gradually declines soon after from approximately 25 weeks of age (Singer, 2006). This means that in many countries, by approximately 72 weeks of age, flocks are considered economically unviable and are slaughtered after approximately 12 months of egg production (Hernandez, 2005) although chickens will naturally live for 6 or more years. In some countries, hens are force moulted to re-invigorate egg-laying. Environmental conditions are often automatically controlled in egg-laying systems (Jonathan, 2011). For example, the duration of the light phase is initially increased to prompt the beginning of egg-laying at 16-20 weeks of age and then mimics summer day-length which stimulates the hens to continue lying all year round; normally, egg production occurs only in the warmer months. Some commercial breeds of hen can produce over 300 eggs a year (Jonathan, 2011). Critics argue that year-round egg production stresses the birds more than normal seasonal production. 
There are two main poultry rearing approach, these includes:

a. $\quad$ Free-range poultry farming allows chickens to roam freely for a period of the day, although they are usually confined in sheds at night to protect them from predators or kept indoors if the weather is particularly bad.

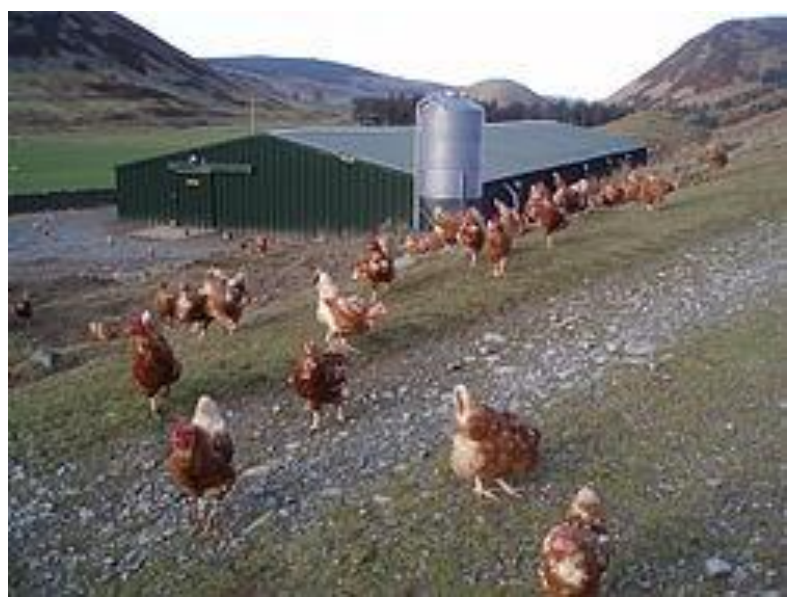

Figure 1: Commercial free range hens

The benefits of free-range poultry farming for laying hens include opportunities for natural behaviours such as pecking, scratching, foraging and exercise outdoors (Jonathan, 2011).

Both intensive and free-range farming have animal welfare concerns. Cannibalism, feather pecking and vent pecking can be common with some farmers using beak trimming as a preventative measure, although reducing stocking rates would eliminate these problems. Diseases can be common and the animals are vulnerable to predators (Sherwin et al., 2010).

b. The majority of hens in many countries are reared in battery cages. These are small cages, usually made of metal in modern systems, housing 3 to 8 hens. The walls are made of either solid metal or mesh, and the floor is sloped wire mesh to allow the faces to drop through and eggs to roll onto an egg-collecting conveyor belt. Water is usually provided by overhead nipple systems, and food in a trough along the front of the cage replenished at regular intervals by a mechanical chain (Adler and Lawler, 2012).

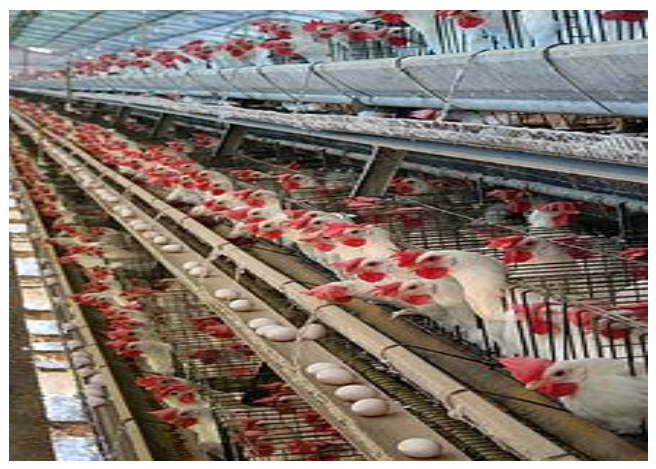

Figure 2: Hens in a battery cage system 
Imianvan A.A., Obi J.C.; Neuro-Fuzzy Supervised Training Algorithm for Varied Chicken Disease Recognition. Journal of Biomedical Engineering and Medical Imaging, Volume 1, No. 6, Dec (2014) , pp 47-55

Majority of diseases affecting poultry bird are usually tied to the respiratory organ of the system which usually interlink with other system such as air sac of the body. The respiratory system are usually are of particular note to poultry bird because of the easy of disease flow and spread (Local Harvest, 2010).

The focal point of this research is geared toward recognizing four common poultry disease utilizing a Neuro-Fuzzy (Neural Network and Fuzzy Logic) Algorithm approach.

\section{Material and Method}

Four common chicken diseases are: (Local Harvest, 2010)

a. Infectious Bronchitis: Infectious Bronchitis is a diseases respiratory based in backyard flock chickens that is relatively common. This condition is also known as "Bronchitis" and a "Cold". This disease is specific to chickens in particular when it comes to types of poultry. The infection may be mild to severe, depending on several circumstances such as the strength of the immunity of the bird, and other conditions present in the environment in which the chicken is located. Chicken diseases symptoms that are present when it comes to this particular illness include, but are not limited to:

P1: Lack of regular food consumption

P2: Liquid discharge in eyes and nostrils

P3: Breathing complications with gasping

P4: Distinguishable chirping sounds

P5: Reduced dramatically egg production

b. Avian Influenza: Many chicken owners have discovered that the chickens in their flocks come down with a condition which is called "Avian Influenza". This is also known as the "Fowl Plague", and the "Flu". While any type of bird may acquire this illness, chicken owners should be concerned because of the fact that it can spread rapidly through flocks. There are many ways that this condition can be transmitted from one chicken to another, making it a large concern when it comes to diseases respiratory in home flocks. These methods include contamination by shoes that can carry it from one location to another, insects, rodents, and even equipment used in chicken coops and the basic care of chickens. Chicken diseases symptoms may include any or all of the following:

P6: $\quad$ Respiratory function distress

P7: $\quad$ Rapid eating habits change

P8: $\quad$ Mild-severe diarrhea

P9: Decrease in egg production rates

P10: Reddish or whitish spot on the legs of the chicken affected

P11: Irritated legs, and wings

c. Mycoplasma Galliseptiu: Mycoplasma Gallisepticum or "Chronic Respiratory Disease" is a disease in respiratory system that many chickens may be affected by. Many may also refer to 
this illness as "Mycoplasmosis" or "Infectious Sinusitis". While poultry such as turkeys and ducks may be affected by this condition, backyard flock chickens are commonly affected as well. Chicken diseases symptoms present with these diseases in respiratory system include:

P12: $\quad$ Swollen sinuses

P13: An obvious sneeze may become apparent

P14: Discharge may be emitted through the nasal cavity

P15: A foamy based discharge may be evident in the eyes

P16: Unusual sounds emitted when taking breaths

d. Fowl Pox: Fowl Pox is another common chicken disease found in backyard flocks. This is known by many names, such as "Chicken Pox" (Not the same as the human version), "Bird Pox", and "Avian Diphtheria". When poultry suffers from these diseases respiratory, there are many chicken diseases symptoms present, such as:

P17: Lesions that reflect that of "warts" often appear on the areas of the body where there are no feathers

P18: Egg production rate is typically hindered

P19: $\quad$ The skin may appear raw, and this may be accompanied by bleeding

P20: Noticeable distress in respiratory function

P21: Congestion in the respiratory system may be experienced

The theory of fuzzy logic provides a mathematical strength to capture the uncertainties associated with human cognitive processes, such as thinking and reasoning. In standard set theory, an object does or does not belong to a set. There is no middle ground. In such bivalent systems, an object cannot belong to both its set and its compliment set or to neither of them. This principle preserves the structure of the logic and avoids the contradiction of object that both is and is not a thing at the same time (Zadeh, 1965). However, fuzzy logic is highly abstract and employs heuristic (experiment) requiring human experts to discover rules about data relationship (Angel and Rocio, 2011).

Fuzzy classification assumes the boundary between two neighboring classes as a continuous, overlapping area within which an object has partial membership in each class (Kuang et al., 2011). Fuzzy logic highlights the significant of most applications in which categories have fuzzy boundaries, but also provides a simple representation of the potentially complex partition of the feature space. (Sun and Jang, 1993 and Ahmad, 2011) Conventional approaches of pattern classification involve clustering training samples and associating clusters to given categories. The complexity and limitations of previous mechanisms are largely due to the lack of an effective way of defining the boundaries among clusters. This problem becomes more intractable when the number of features used for classification increases (Christos and Dimitros, 2008).

Artificial Neural Networks (ANNs) constitute a class of flexible nonlinear models designed to mimic biological neural systems. An ANN is a mathematical model or computational model based on biological 
Imianvan A.A., Obi J.C.; Neuro-Fuzzy Supervised Training Algorithm for Varied Chicken Disease Recognition. Journal of Biomedical Engineering and Medical Imaging, Volume 1, No. 6, Dec (2014) , pp 47-55

neural networks (Gutiérrez, 2011), as an interconnected group of artificial neurons, which carries out computation using a connectionist approach. Typically, a biological neural system consists of several layers, each with a large number of neural units (neurons) that can process the information in a parallel manner. The models with these features are known as ANN models (Robert, 2000). ANNs have been widely applied to solve many difficult problems in different areas, including pattern recognition (matching), signal processing, language learning, electronic medical record processing, tele-diagnosis and computer networking (Robert, 2000). Neural network utilize dataset. The data set is divided into three distinct sets: training, testing and validation sets. The training set is the largest set and is used by neural network to learn patterns present in the data. The testing set is used to evaluate the generalization ability of a supposedly trained network. A final check on the performance of the trained network is made using validation set. Learning methods in neural networks can be broadly classified into three basic types Supervised, unsupervised and reinforced learning (Diogo et al. 2008).

Supervised learning is the machine learning task of inferring a function from supervised training data. The training data consist of a set of training examples. In supervised learning, each example is a pair consisting of an input object (typically a vector) and a desired output value (also called the supervisory signal). A supervised learning algorithm analyzes the training data and produces an inferred function, which is called a classifier (if the output is discrete) or a regression function (if the output is continuous).

Unsupervised learning studies how systems can learn to represent particular input patterns in a way that reflects the statistical structure of the overall collection of input patterns. By contrast with Supervised Learning or Reinforcement Learning, there are no explicit target outputs or environmental evaluations associated with each input; rather the unsupervised learner brings to bear prior biases as to what aspects of the structure of the input should be captured in the output. Unsupervised learning is important since it is likely to be much more common in the brain than supervised learning (Benedetti et al., 2005).

Reinforcement learning, one of the most active research areas in artificial intelligence, is a computational approach to learning whereby an agent tries to maximize the total amount of reward it receives when interacting with a complex, uncertain environment. Reinforcement learning provides a clear and simple account of the key ideas and algorithms. Their target ranges from the history of the field's intellectual foundations to the most recent developments and applications. The only necessary mathematical background is familiarity with elementary concepts of probability (Richard and Andrew, 2011).

The four most widely used neural networks are the feed-forward networks and recurrent or interactive (feedback) networks, kohonen's self-organizing network, Adaptive resonance Theory (ART) and Counter propagation network are others (Chakraborty, 2010).

Feed-forward ANNs allow signals to travel one way only; from input to output. There is no feedback (loops) i.e. the output of any layer does not affect that same layer. They are extensively used in pattern recognition (Chakraborty, 2010).

This multi-layered structure of a feed-forward network is designed to function as a biological neural system. The input units are the neurons that receive the information (stimuli) from the outside environment and pass them to the neurons in a middle layer (i.e., hidden units). These neurons then 
transform the input signals to generate neural signals and forward them to the neurons in the output layer. The output neurons in turn generate signals that determine the action to be taken. It is important to note that all information from the units in one layer is processed simultaneously, rather than sequentially, by the units in an "upper" layer (kuan and white, 1994).

Feedback Network or Recurrent Neural Networks: Feedback networks can have signals travelling in both directions by introducing loops in the network. Feedback networks are dynamic; their 'state' is changing continuously until they reach an equilibrium point. They remain at the equilibrium point until the input changes and a new equilibrium needs to be found (Chakraborty, 2010).

Kohonen's Self-Organizing Network is a two-layer, feed-forward network (Beale and Jackson, 1990 and Dayhoff, 1990).The first is an input layer and the second is a grid or map arranged in a one or twodimensional array. The second layer is known as a competitive layer. Incoming patterns are classified by the nodes that they activate in the competitive layer. Similarities among patterns are mapped into closeness relationships on the competitive layer. After training, the pattern relationships and groupings are observed from this layer.

Adaptive Resonance Theory (ART) is an unsupervised, competitive learning algorithm (Beale and Jackson, 1990). It is a two-layer network arranged in feedback and feed-forward connection. The layers have different functions, unlike the Multilayer or Kohonen networks. The first layer can be either an input or a comparison layer and the second layer can be either an output or a recognition layer. Both are interchangeable during training.

\subsection{Method: The Proposed Neuro-Fuzzy Supervised Training Algorithm for Varied Chicken Disease Recognition}

The proposed Algorithm imbibes artificial intelligence techniques in tying the symptoms of chicken disease to the differential recognition of four classes thereby establishing a conclusive boundary. Unlike the current approaches, in which success or failure are based on the wills and experiences of relevant personnel designing and administrating the approach in other to elicit relevant recognition points. The forecasted algorithm is artificial intelligence based; therefore success and failure are not dependent on human intuitions, but success is closely linked to the tuned-up components of the systematic implemented approach. The Algorithm is depicted on Figure 1

INPUT:

Types of Chicken Disease (Infectious Bronchitis, Avian Influenza, Mycoplasma Galliseptiu, and Fowl Pox,)

No. of Symptoms (P1, P2, P3..., Pn) $=20$

P; Fuzzy parameters (Symptoms Codes)

Degree of membership function

$\geq 0.50=$ High degree membership function (serious)

$<0.50=$ Low degree Membership Function (minor)

Fuzzy predefined Rules

More than five symptoms $=$ Diagnose with a class of Chicken disease

Exactly four symptoms $=$ Might be diagnose with a Chicken disease

Three symptoms and below $=$ Not diagnose with a Chicken disease 
Imianvan A.A., Obi J.C.; Neuro-Fuzzy Supervised Training Algorithm for Varied Chicken Disease Recognition. Journal of Biomedical Engineering and Medical Imaging, Volume 1, No. 6, Dec (2014) , pp 47-55

// INITIALIZATION

1. Randomly pick a Chicken $K$;

2. Save identification (recognition) Result in Knot;

// Loop till terminal point

3. For $\mathrm{P}=1$ to $\mathrm{n}$ do;

// INFECTIOUS BRONCHITIS

4. Recognize for Infectious Bronchitis

5. If symptoms are $\mathbf{P 1 - P 5}$, symptoms membership are serious, and symptoms $\geq$ five THEN infectious bronchitis;

6. Else if

7. Might be Infectious Bronchitis; (symptoms = four);

8. Else

9. Not Infectious Bronchitis (symptoms $\leq$ three);

// AVIAN INFLUENZA

10. Recognize for Avian Influenza;

11. If symptoms are $\mathbf{P 6 - P 1 1}$, symptoms membership are serious, and symptoms $\geq$ five THEN Avian Influenza;

12. Else if

13. Might be Avian Influenza (symptoms = four);

14. Else

15. Not Avian Influenza (symptoms $\leq$ three);

//MYCOPLASMA GALLISEPTIU

16. recognize for Mycoplasma Galliseptiu;

17. If symptoms are $\mathbf{P} \mathbf{1 2}-\mathbf{P} \mathbf{1 6}$, symptoms membership are serious, and symptoms $\geq$ five THEN Mycoplasma Galliseptiu;

18. Else if

19. Might be Mycoplasma Galliseptiu (symptoms = four);

20. Else

21. Not Mycoplasma Galliseptiu (symptoms $\leq$ three);

//Fowl Pox

22. recognize for Fowl Pox;

23. If symptoms are $\mathbf{P} \mathbf{1 7}-\mathbf{P 2 1}$, symptoms membership are serious, and symptoms $\geq$ five THEN Fowl Pox;

24. Else if

25. Might be Fowl Pox (symptoms = four);

26. Else

27. Not Fowl Pox (symptoms $\leq$ three);

//Save results in Knot;

28. Return chicken disease result for chicken $\mathrm{K}$

Figure 1: The Proposed Neuro-Fuzzy Supervised Training Algorithm for Varied Chicken Disease Recognition

\section{Implementation and Discussion}

The implementation of our result was dual fold; the neural training dataset was handled conveniently utilizing Matrix Laboratory (MATLAB) which serves as our simulation tool in achieving the our results because of its interactive environment for algorithm development, data visualization, data analysis, and numerical approach which was relevant to our numerical dataset which was more appropriate than with 
spread-sheets or traditional programming languages, such as $\mathrm{C} / \mathrm{C}++$ or Java. MATLAB was essential in validating some of the if-then rules concept with the training algorithm. The design alternatives was distributed and visualized in various concept utilizing MATLAB tools. The debugging tools in MATLAB were useful in identifying underlying errors and eliminating them. After pruning the dataset utilizing MATLAB the algorithm was fully implemented utilizing Hypertext Preprocessor (PHP), which served as the language of implementation. PHP provide the languages for writing the dynamic web-pages which enable any veterinary personal to access the system online retrieved relevant information in determining the relevant diseases.

\section{Discussion}

The implemented algorithm provides an interactive base in determining varied chicken disease objectively as opposed to the subjective approach which is achievable utilizing the subjective approach. The result was satisfactory having been able to distinctively recognize several chicken disease and subsequently classified into varied classes.

\section{Conclusions}

This paper has demonstrated the practical application of neuro- fuzzy supervised training algorithm in the agricultural sector by providing a means of recognizing varied chicken disease.

\section{REFERENCES}

[1] Adler J.; Lawler A. (2012), How the Chicken Conquered the World, Smithsonian, Retrieved May 27, 2012.

[2] Ahmad H. (2011), Fuzzy approach to Likert Spectrum in Classified levels in surveying Researches retrieved http://www.tjmcs.com.

[3] Angel C. and Rocio R. (2011), Documentation management with Ant colony Optimization Metaheuristic: A Fuzzy Text Clustering Approach Using Pheromone trails, retrieved from soft computing in Industrial applications, Advances in intelligent and soft Computing, vol. 96, 2011, 261-70, DOI: 10.1007/978-3-642-20505-1_23

[4] Benedetti S., Saverio M., Anna G. S. and Gian L.M. (2005), Electronic nose and neural network use for the classification of honeypot, retrieved from citeseerx.ist.psu. Edu/viewdoc /download?doi=10.1.1.128.pdf

[5] Beale R. and Jackson I. (1990), Neural Computing: An Introduction, dl.acm.org/citation.cfm?id=121342.

[6] Chakraborty R.C. (2010), Soft computing-Introduction: Soft-computing Lecture 1-6, notes, retrieved from http://myreaders.inro

[7] Christos S. and Dimitros S. (2008) Neural Network, retrieved from http://www.docstoc.com/docs/15050/neural-networks. 
Imianvan A.A., Obi J.C.; Neuro-Fuzzy Supervised Training Algorithm for Varied Chicken Disease Recognition. Journal of Biomedical Engineering and Medical Imaging, Volume 1, No. 6, Dec (2014) , pp 47-55

[8] Dayhoff, J.E. (1990), Neural Network Architecture: An Introduction, retrieved from https://catalyst.library.jhu.edu/?q=\%22Dayhoff\%2C+Judith.

[9] Diogo F. P., Flávio R.S. O. and Fernando B. L. N (2008), Multi-objective abilities in the Hybrid Intelligent Suite for decision support, retrieved from http://ieeexplore. ieee.org/xpl/freeabs_all.

[10] Grandin T.; Johnson, C. (2005), Animals in Translation, New York, NY: Scribner. Pp. 183, ISBN 07432-4769-8.

[11] Gutiérrez P.A. (2011), Hybrid Artificial Neural Networks: Models, retrieved online from http:// dl.acm.org/citation.cfm?id=20233.

[12] Hernandez N., (2005), Advocates Challenge Humane-Care Label on Md. Eggs. Washington Post, Retrieved 2009-07-30.

[13] Jonathan S. (2011). Delaware business: Chicken companies feeling pinch as corn prices soar, News Journal (Gannett), Delaware-Online. OCLC 38962480. Retrieved 10 April 2011.

[14] Kuan C. M. and White H. (1994), Artificial neural networks: An econometric perspective, Econometric Reviews, Vol.13, Pp.1-91 and Pp.139-143.

[15] Kuang Y. H.; Ting-H. C. and Ting-Cheng Chang (2011), Determination of the threshold value $\beta$ of variable precision rough set by fuzzy algorithms, retrieved from http://www.sciencedirect.com/science/article/pii/S0888613X11000831.

[16] LocalHarvest, (2010), Common Chicken Diseases and Symptoms in Backyard Flocks http: //www.localharvest.org/blog/26992/entry/common_chicken_diseases_and_symptoms.,

[17] Richard S. S. and Andrew G. B. (2011), Reinforcement learning: an Introduction, retrieved from http://books.google.com/books/about/Reinforcement_l.

[18] Robert F. (2000), Introduction to Neuro-Fuzzy Systems: Advances in Soft Computing Series, Springer-Verlag, Berlin/Heidelberg, Germany.

[19] Sherwin, C.M., Richards G.J and Nicol C.J. (2010), A comparison of the welfare of layer hens in four housing systems in the UK. British Poultry Science, 51(4): 488-499.

[20] Singer P. (2006), In Defence of Animals, Wiley-Blackwell, Pp. 176, ISBN 1-4051-1941-1.

[21] Sun C.T. and Jang J.S. (1993) A neuro-fuzzy classifier and its applications, in: Proc. IEEE Int. Conference on Neural Networks, San Francisco, pp.94-98.

[22] Zadeh L.A. (1965), Fuzzy sets, Journal of Information and Control”, Vol.8, pp.338-353. 